INPLASY

PROTOCOL

To cite: Hu et al. Different Chinese patent medicine therapies for migraine $A$ protocol for systematic review and network meta-analysis. Inplasy protocol 2020120010. doi:

10.37766/inplasy2020.12.0010

Received: 02 December 2020

Published: 02 December 2020

Corresponding author:

Chunhua Huang

1072065006@qq.com

Author Affiliation:

Affiliated Hospital of Jiangxi

University of

Traditional Chinese Medicine;

Xinyu Hospital of Traditional

Chinese Medicine

Support: None.

Review Stage at time of this submission: Preliminary searches.

Conflicts of interest:

None.

\section{Different Chinese patent medicine therapies for migraine A protocol for systematic review and network meta- analysis}

\author{
Hu, L1; Chen, F2; Xiao, W3; Liu, L4; Huang, C5.
}

Review question / Objective: Which Chinese patent medicine is the most effective and safe treatment for migraine.

Condition being studied: Migraine is a neurovascular disease with a high clinical incidence. Female More than men, the disease is a kind of primary headache, often recurrent, headache Intense, accompanied by nausea, vomiting, phonophobia, photophobia and other symptoms. When migraine attacks, the patient Often unable to carry out daily activities due to pain, which seriously affects the quality of life of patients the amount. At present, there is no radical treatment method in clinical practice, only drug control is used. Control the condition, but due to the recurrence of the disease, the long-term use of western medicine has more side effects, so it is necessary to find Seek safer and more effective treatments.

INPLASY registration number: This protocol was registered with the International Platform of Registered Systematic Review and Meta-Analysis Protocols (INPLASY) on 02 December 2020 and was last updated on 02 December 2020 (registration number INPLASY2020120010).

\section{INTRODUCTION}

Review question / Objective: Which Chinese patent medicine is the most effective and safe treatment for migraine.
Condition being studied: Migraine is a neurovascular disease with a high clinical incidence. Female More than men, the disease is a kind of primary headache, often recurrent, headache Intense, accompanied by nausea, vomiting, 
phonophobia, photophobia and other symptoms. When migraine attacks, the patient Often unable to carry out daily activities due to pain, which seriously affects the quality of life of patients the amount. At present, there is no radical treatment method in clinical practice, only drug control is used. Control the condition, but due to the recurrence of the disease, the long-term use of western medicine has more side effects, so it is necessary to find Seek safer and more effective treatments.

\section{METHODS}

Participant or population: Randomized controlled trial.

Intervention: The experimental group used proprietary Chinese medicines, including Toutongning Capsules, Yangxue Qingnao Granules, Naoxintong Capsules, Tianma Gouteng Granules.

Comparator: The control group used Western medicine alone.

Study designs to be included: Patients diagnosed with migraine in accordance with internationally recognized diagnostic criteria have clear curative effect standards, regardless of age, race, gender, and source of cases.

Eligibility criteria: Based on the RCTs of different Chinese patent medicines for migraine, the language is limited to Chinese and English.

Information sources: The two reviewers first screened them independently according to the literature inclusion and exclusion criteria, and then cross-checked them. In case of disagreement, the third reviewer can make a decision. Establish a document information extraction table in EXCEL, and extract information including: author, publication time, number of cases, allocation method, intervention measures, treatment course and outcome indicators, etc.

Main outcome(s): The main outcome indicators include total effective rate, visual analog pain scale (VAS) score, and the secondary outcome indicators include analgesic effect evaluation indicators, quality of life scale.

Quality assessment / Risk of bias analysis: Two reviewers independently conducted a bias risk assessment of the final included literature in this study with reference to the Cochrane reviewer bias risk assessment tool, including selection bias, implementation bias, measurement bias, follow-up bias, reporting bias, and other source biases. Evaluation results are evaluated in terms of "high risk", "low risk" and "unclear risk".

Strategy of data synthesis: Computer search for PubMed, Cochrane library, Web of Science, Embase, SinoMed, CNKI, Wanfang database, VIP. The search terms are: "Toutongning capsule", "yangxue qingnao granule", "naoxintong capsules" ", "Tianma Gouteng granules", "Migraine", "headache", "Primary headache". The search time is from the establishment of the database to November 18, 2020. All retrieval strategies used multiple retrievals in the form of subject terms + free words, and manually retrieved relevant references.

Subgroup analysis: If the $\mathbf{I 2}>\mathbf{5 0 \%}$, we will explore sources of heterogeneity by subgroup analysis and meta-regression, the STATA software will be used for subgroup analysis and meta-regression analysis according to the characteristics of the test.

Sensibility analysis: Sensitivity analysis will be completed by changing the effect model or removing 1 study at a time to investigate the influence of a single study on the overall pooled estimate.We will evaluate the robustness of the meta-analysis results through sensitivity analysis, and exclude such as small-sample trials and low-quality trials to explore the impact of trial quality on efficacy estimates.

Country(ies) involved: China. 
Keywords: Chinese patent medicine;

network meta-analysis, protocol, migraine.

Contributions of each author:

Author 1 - Liangen Hu.

Author 2 - Feng Chen.

Author 3 - Wei Xiao.

Author 4 - Liting Liu.

Author 5 - Chunhua Huang. 